\title{
International Space Station Urine Monitoring System Functional Integration and Science Testing
}

\author{
Branelle R. Rodriguez ${ }^{1}$ and James Lee Broyan, $\mathrm{Jr}^{2}$ \\ NASA Lyndon B. Johnson Space Center, Houston, Texas, 77058
}

\begin{abstract}
Exposure to microgravity during human spaceflight needs to be better understood as the human exploration of space requires longer duration missions. It is known that long term exposure to microgravity causes bone loss. Measuring the calcium and other metabolic byproducts in a crew member's urine can evaluate the effectiveness of bone loss countermeasures. The International Space Station (ISS) Urine Monitoring System (UMS) is an automated urine collection device designed to collect urine, separate the urine and air, measure the void volume, and allow for syringe sampling. Accurate measuring and minimal cross-contamination is essential to determine bone loss and the effectiveness of countermeasures. The ISS UMS provides minimal cross-contamination $(<0.7 \mathrm{~mL}$ urine) and has volume accuracy of $\pm 2 \%$ between 100 to $1000 \mathrm{~mL}$ urine voids. Designed to provide a non-invasive means to collect urine samples from crew members, the ISS UMS operates inline with the Node 3 Waste and Hygiene Compartment (WHC). The ISS UMS has undergone modifications required to interface with the WHC, including material changes, science algorithm improvements, and software platform revisions. Integrated functional testing was performed to determine the pressure drop, air flow rate, and the maximum amount of fluid capable of being discharged from the UMS to the WHC. This paper will detail the results of the science and the functional integration tests.
\end{abstract}

\section{Nomenclature}

$\begin{array}{ll}\text { ACY } & =\text { Russian Toilet } \\ \mathrm{AM} & =\text { Avionics Module } \\ { }^{\circ} \mathrm{C} & =\text { degrees Celsius } \\ \mathrm{ddH} \mathrm{H}_{2} \mathrm{O} & =\text { distilled water } \\ \mathrm{ISS} & =\text { International Space Station } \\ \mathrm{L} & =\text { Liter } \\ \mathrm{min} . & =\text { Minute } \\ \mathrm{mL} & =\text { mili-Liter } \\ \mathrm{mm} & =\text { mili-meter } \\ \mathrm{MM} & =\text { Mechanical Module } \\ \mathrm{NASA} & =\text { National Aeronautics and Space Administration } \\ \mathrm{S} & =\text { second } \\ \mathrm{SSC} & =\text { Space Station Computer } \\ \mathrm{STS} & =\text { Space Transportation System } \\ \mathrm{UCD} & =\text { Urine Collection Device } \\ \mathrm{UMS} & =\text { Urine Monitoring System } \\ \mathrm{US} & =\text { United States } \\ \text { WCS } & =\text { Waste Collection System } \\ \text { WHC } & =\text { Waste and Hygiene Compartment }\end{array}$

${ }^{1}$ UMS Project Manager, Crew \& Thermal Systems Division, M/S: EC3, not AIAA affiliated..

${ }^{2}$ Habitability Hardware Group Lead, Crew \& Thermal Systems Division, M/S: EC3, not AIAA affiliated.. 


\section{Introduction}

The detailed design considerations and operation of the Urine Monitoring System (UMS) have been previously described $^{1}$ but major characteristics are summarized here. The monitoring of urine is an important aspect of crew member health. Monitoring of both the total urine volume and chemical constituent concentrations on-orbit during a mission can provide early detection of health problems and allow the determination of the effectiveness of zero-g countermeasures, such as nutrition, prescription medications, and exercise routines. Collection of medical urine samples is currently accomplished with Urine Collection Device (UCD) ${ }^{1}$ bags that require an adapter for either males or females, and are crew time intensive, prone to unintentional releases of urine into the cabin, and have received negative crew feedback. The UMS, Figure 1, is an automated urine collection device that collects individual crew member micturitions, separates it from the air stream, measures total urine void volume, allows for syringe sampling, and transfers the urine to the downstream waste collection and processing hardware. Functionally, the UMS utilizes the Russian Toilet (ACY) ${ }^{2}$ fan separator in the Waste and Hygiene Compartment (WHC) to entrain urine into the UMS and then onto the WHC system.

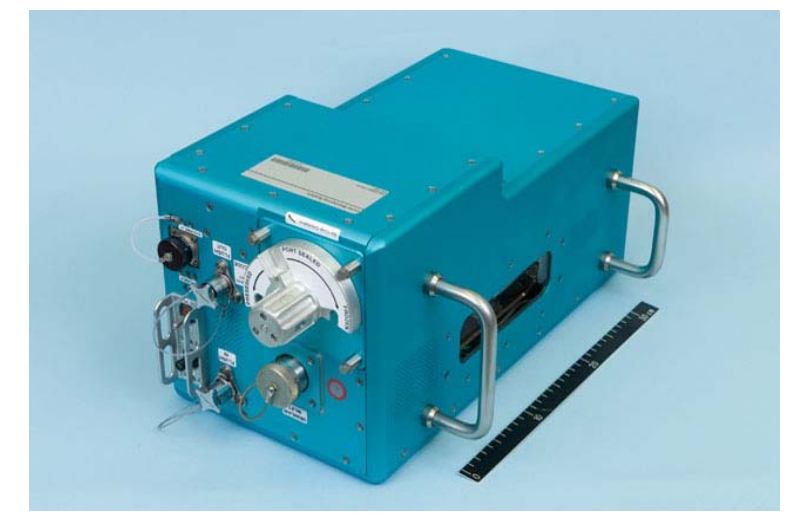

Figure 1: Urine Monitoring System Mechanical Module

The paper's intent is to describe significant changes required to address hardware/design modifications, hardware integration development tests, and on-orbit validation and operation planning. The Urine Monitoring System was originally designed for use with the Space Shuttle Waste Collector System (WCS) with future plans for minor modifications for use on International Space Station (ISS) with an Extended Duration Orbiter derived WCS. However, due to programmatic constraints, the UMS Space Shuttle mission was demanifested and the original UMS was modified for ISS operations. For use on ISS, the system will interface with the WHC located in the Node 3 element. Since UMS' initial development, the WHC architecture changed from a United States (US) WCS to a Russian toilet (ACY) $)^{3}$. The combination of the interface changes required modifications to the UMS separator bowl, internal plumbing, science algorithms, operation software, integration testing with the Russian ACY, and changes to the physical installation and validation on-orbit.

\section{UMS Detailed Description}

The major UMS science requirements include micturation volume and accuracy, minimal cross-contamination, and the ability to perform sample collection. The UMS measures individual micturation volumes with an accuracy requirement of $\pm 10 \%$ for void volumes between 30 and $100 \mathrm{~mL}$, and $\pm 2 \%$ for void volumes above $100 \mathrm{~mL}$ and up to $1000 \mathrm{~mL}$. The system allows for multiple samples of individual micturation volumes with $10 \mathrm{~mL}$ syringes. Specifically, the UMS allows one syringe to be drawn for 30-60 mL voids, two syringes for $60-100 \mathrm{~mL}$ voids, three syringes for $100-500 \mathrm{~mL}$ voids, and four syringes for $500-1000 \mathrm{~mL}$ voids. To meet the cross-contamination requirement, the UMS is designed to maintain $\leq 0.7 \mathrm{~mL}$ of urine transfer between subsequent crew member micturations, and a $\leq 5.0 \mathrm{~mL}$ of residual fluid between subsequent uses.

The UMS has three physical connections to the WHC front close-out panel; power, flush water, and urine/air discharge, as illustrated in Figure 2. A urine/air discharge line internal to the WHC provides the connection from the WHC front close-out panel to the ACYs urinal hose connection, see Figure 3. During nominal operations, the airflow entrains the crew member's urine stream as a two phase urine/air mixture through the UMS urinal funnel and hose. The mixed flow immediately passes through a replaceable mesh screen filter to remove large debris that could potentially reduce the life of the rotary separator. The mixed flow then enters the rotary separator where the urine is directed, through centrifugal force, to the separator bowl's outer wall where it accumulates in an annular 
fluid ring until the void is complete. The UMS rotary separator design utilizes a rotating bowl and paddles, rotating at the same speed, in order to produce near solid body fluid rotation. This greatly reduces the turbulence and foaming within the unit and facilitates accurate pressure measurements. Multiple pressure measurements of the urine void are taken to determine its volume. Once all measurements have been taken, the drain solenoid valve (SOL4 in Figure 2) is then opened briefly to fill the drain line to the sample port assembly. The crew member can then collect samples via syringes or opt not to collect samples. After sample collection is completed, the drain solenoid valve is then opened to drain the residual urine from the separator. The drained urine is recombined with the airflow before delivery to the WHC/ACY. This enables the WHC/ACY to receive the same two phase urine/air flow and operate in a similar manner with or without the UMS connected.

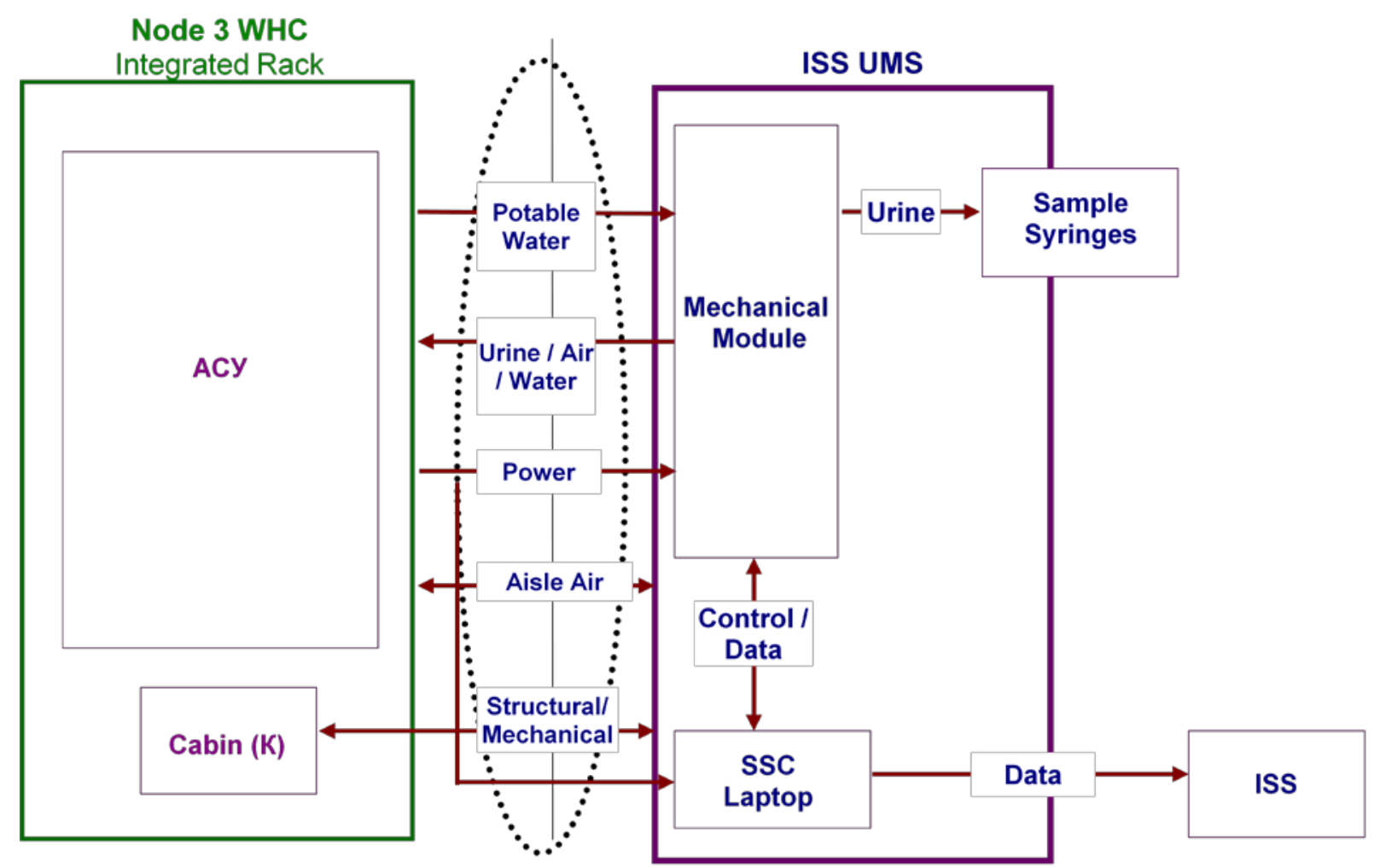

Figure 2: UMS to WHC Block Diagram

After the urine/air mixture is drained to the WHC/ACY, the UMS is then supplied with water to reduce residual urine contamination. Water flow is provided by the pressurized vehicle potable water supply through two redundant shutoff solenoid valves. Three orifices distribute the flow between parallel paths within the UMS. This provides flushing of the urinal hose and separator bowl, and prevents dispersal of urine into the small pressure sensing lines. The flush water is then drained through the drain solenoid valve to the WHC/ACY. Upon completion, the UMS enters a standby mode until used by the next crew member. 


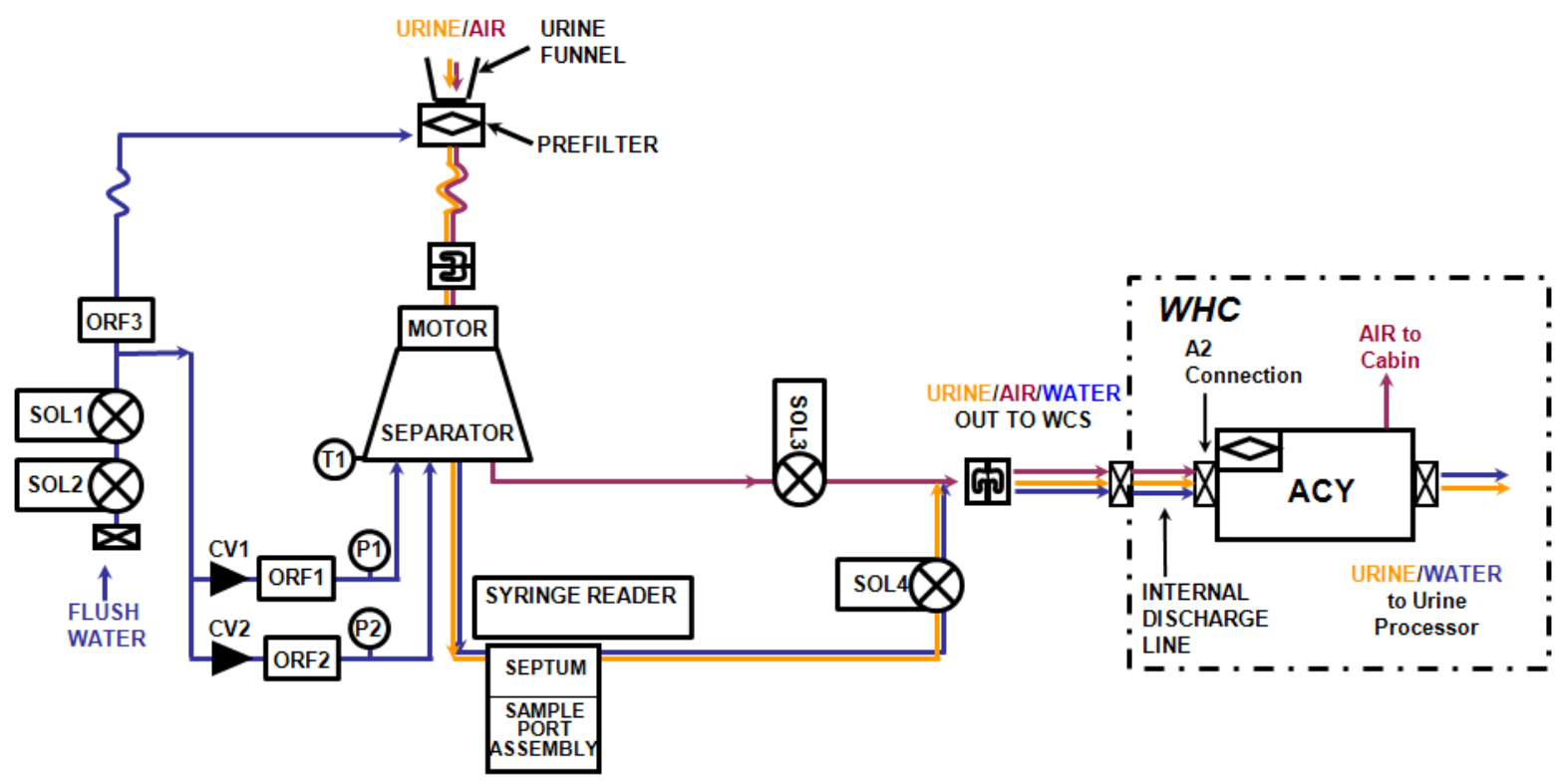

Figure 3: UMS flow schematic illustrating major components and flow of air, urine, and flush water.

\section{A. Software Modifications}

The software application was originally written for UMS in C programming software and imbedded into the Avionics Module (AM) of the UMS. The AM did not allow for future on-orbit adjustability and had obsolete parts creating challenges during ISS integration. Therefore, the AM was removed from the UMS design and replaced with a Space Station Computer (SSC) Laptop. The SSC Laptop is a ThinkPad T61P Laptop and is connected to the UMS through the electrical interface cable. Additionally, the UMS software was modified to operate on a JAVA platform utilizing $\mathrm{C}++$ programming software to improve operating efficiency.

The purpose of the UMS flight software is to control and manage UMS on-orbit operations. Through an SSC laptop, the software will provide activation and control to the Mechanical Module (MM), provide a crew user interface, and provide for subsystems data storage. The software is capable of controlling the UMS internal components and hardware, simplifying human and machine interfaces, data logging and storage, and system health/status monitoring.

The UMS system has the following on-orbit (flight) operational modes: Micturition (nominal) Mode, Validation Mode, and Maintenance Mode. The Micturition Mode is the normal mode for the UMS system. During this mode the SSC Laptop displays messages which guide the crew member through the nominal operational procedures. Validation Mode provides the capability to perform on-orbit validation of the UMS. This is a sequence of operations during which the crew member adds known volumes of liquid to the UMS and the software executes a comparison of the actual value(s) to the measured value(s). The software logs the results for post flight analysis. Cleaning/Maintenance Mode provides for the nominal on-orbit cleaning and maintenance of the system, including the ability to provide a manual flush of vehicle water.

\section{Functional Integration Testing}

The UMS was originally designed to be compatible with the Shuttle WCS fans' flow rates of $\sim 280-380 \mathrm{~L} / \mathrm{min}$. The air is used for entrainment of urine from the crew member at the urinal funnel and conveying it to the UMS. As the air flows through UMS it is directed through several turns and coalescor disks (rotating screens that remove small liquid drops from the airstream) creating an air resistance of $\sim<175 \mathrm{~mm}$ of water pressure drop. The UMS pressure drop is a complex function of the urine flow rate into the urinal hose, the UMS separator rotational speed, and the total urine void volume. The fan air flow rate is not fixed but is dependent on the total system resistance according to the individual fan's rotor and motor characteristics. Generally, air flow is reduced as system resistance increases until the fan is no longer able to overcome the system resistance and flow drops to approximately zero. Liquid flow from the UMS is another unique characteristic because urine is collected and held in the UMS separator. The liquid (urine and/or flush water) is measured at set liquid levels and separator speeds and pumped out at variable 
times depending on urine void volume and crew sampling requirements. The air flow is blocked during UMS pressure measurements and during the drain portion of the cycle. The UMS utilizes the fan's suction capacity to 'vacuum' residual liquid from the bowl during draining to minimize liquid carry over between crew uses. Operating the UMS on ISS required the integration to the WHC which utilizes the Russian ACY fan separator.

The Russian ACY system was originally designed without the air restriction introduced by the UMS integration. The ACY separator is controlled by several air/liquid pressure sensors and electrical timing circuits. The correct operation of the pressure switches is required to prevent separator flooding. The correct operation of the electrical timing circuits is required to allow the separator to drain without faults. The variable timing nature of UMS fluid discharges, particularly at large void volumes raised concern of UMS compatibility with the ACY. The ACY fan nominally operates at an air flow rate of $250 \pm 50 \mathrm{~L} / \mathrm{min}$.

To reduce risk, a physical integration test was arranged to ensure the pressure drop, air flow rate, and the maximum amount of fluid capable of being discharged from the UMS to the ACY resulted in acceptable performance for both units. Integration tests were performed in Moscow, Russia in September 2009. The UMS engineering unit which consisted of flight-like separator/fluid components and electronics was utilized. The ACY ground unit which consisted of flight-like fluid components, fan, and electronics was used. The ACY ground unit is known to emulate on-orbit performance and is normally used for investigation of on-orbit problems. There were several ground hardware challenges that were solved with innovative suggestions from our Russian colleagues. The final ground setup between the two units was a high fidelity representation of how the two units would be connected on orbit.

The UMS effluent hose was connected at a 'Y-connector' plumbing location, designated 'A2', immediately below the ACY urinal hose and particulate filter. This A2 connection is currently unused on ISS. Air pressure readings were recorded at several UMS and plumbing locations (points $\mathrm{A}, \mathrm{B}, \mathrm{C}, \mathrm{D}$, and E) depending on the individual test locations, see Figure 3. Air speed/flow rate was also recorded with a hot wire anemometer at points C and D and with a digital orifice plate at location E. The UMS and ACY were initially operated independently to establish normal baseline operations with and without liquids. For purposes of pressure drop and liquid flow tests, distilled water was used. Water was manually introduced into either point $\mathrm{C}$ or point $\mathrm{D}$ at $\sim 10-15 \mathrm{~mL} / \mathrm{s}$.

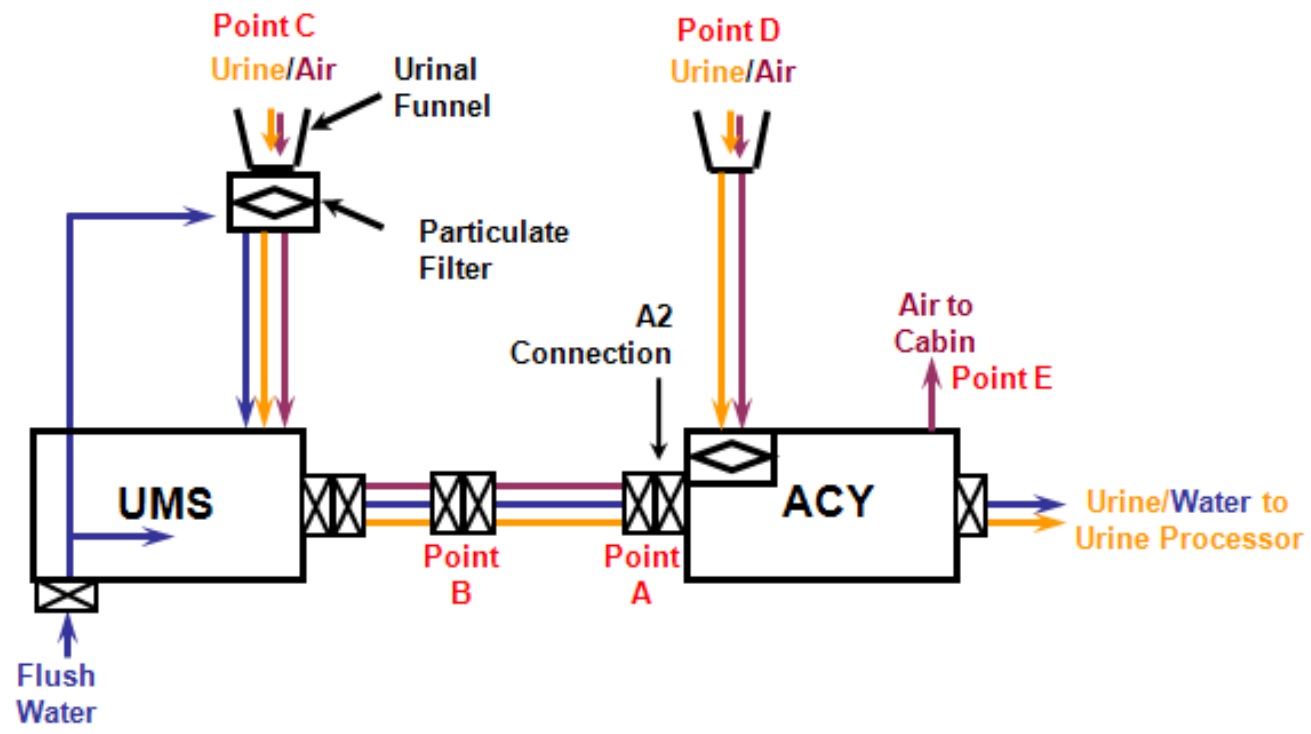

Figure 4: UMS and ACY integration schematic

A series of fluid tests were conducted by introducing water volumes to emulate urine voids. A typical test was conducted by introducing water into the UMS funnel (point C). The ACY funnel (point B) was capped to prevent air intake as would occur during on-orbit operations. The UMS collected the fluid in its separator, performed measurements, and then pumped the fluid (two phase liquid/air flow) to the ACY through tubing points B and A. The ACY rotary separator separated the fluid and delivered it to a waste water container. The separated air exited 
the ACY through its fan and odor filter (point E). Eight different fluid volumes (20 to $1100 \mathrm{~mL}$ ) were tested multiple times to represent the different operational modes and the physical urine discharge range of a single void.

The results are summarized in Figure 4. The average air flow and fan suction pressure during the micturation phase remained relatively constant until very large void volumes. This indicates that the UMS flow restrictions do not significantly impact urine collection capabilities. The air flow decrease at $1100 \mathrm{~mL}$ is expected. At very high void volumes, the UMS separator's interior air core decreases dramatically and purposely causes an air restriction to cause liquid overflow to the air outlet to protect the separator bearings. The variation in total run time is graphed to illustrate the variable nature of UMS operations. The variation is partly due to increased sampling for higher volumes and partly due to the need to reduce separator speed to stay within pressure sensor full scale limitations and motor torque requirements. At $1100 \mathrm{~mL}$ the time decreases due to reduced science measurements and sampling.

In addition to single operation cycles of the UMS-ACY, repeated cycles were performed to represent the crew wake period when crew members may be waiting to use the WHC. It was assumed the average crew void volume was $350 \mathrm{~mL}$; and it was assumed one minute of elapsed time between ACY shutdown and initiation of the next cycle. Two repeated cycles of $350 \mathrm{~mL}$ voids introduced into UMS point $\mathrm{C}$ were conducted, with a one minute interval between cycles. A worst case cumulative run time was defined as a combination of UMS purge (conducted after 30 days of storage), a $350 \mathrm{~mL}$ void introduced into the UMS at point $\mathrm{C}$, and a subsequent $350 \mathrm{~mL}$ void introduced into the ACY at point D. For all tests the UMS and ACY hardware and electronics performed nominally without errors or fault flags. This indicates that the ACY was able to accept $1100 \mathrm{~mL}$, which is above the maximum UMS fluid volume, and the periodic $\sim 30$ second blockages of the airflow to make UMS pressure measurements and drain residual liquid.

Separately, the integrated safety and hazard assessments were reviewed and minor document modifications were recommended for incorporation. The joint US and Russian interpretation of the testing was that the UMS and ACY were capable of being successfully integrated and operated on ISS.

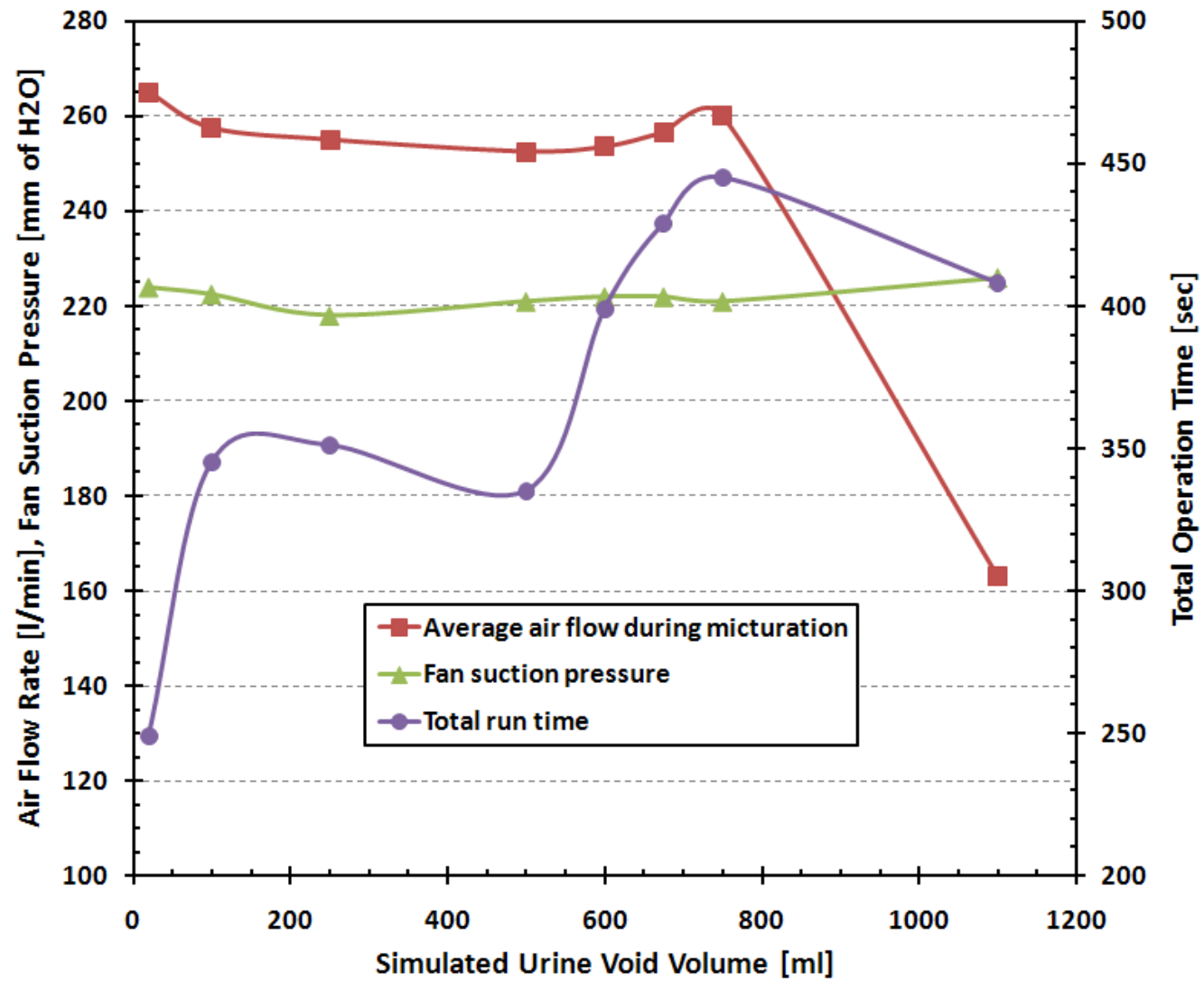

Figure 5: Representative airflow and pressure data from UMS and ACY integration tests 


\section{Science Testing}

\section{A. On-Orbit Validation}

Since urine volume and constituents do vary significantly through a typical day, it is necessary to collect and store samples for a 24-hour period. Nominal on-orbit operations would consist of 2 crew members using UMS for three consecutive days. During use, the UMS must accurately measure the volume of each crew member's micturation, as well as avoid cross-contamination between crew members.

A validation sequence was defined during ground based testing and analysis that will allow confirmation that the UMS is operating as designed on-orbit. This validation sequence consists of fourteen different volume segments designed to measure accuracy, cross-contamination, and residual fluid. Each validation segment consists of a set of syringes holding a specific concentration of pseudo urine or distilled water. The pseudo urine varies between a high, average or low concentration of chemical constituents normally present in urine. High concentrated pseudo urine has a specific gravity range of 1.029 to 1.031 , average concentrated pseudo urine has a specific gravity range from 1.019 to 1.021, and low concentrated pseudo urine has a specific gravity range of 1.009 to 1.011 . The validation segments alternate between the pseudo urine and distilled water. This allows for cross-contamination between successive users to be tested. A potable flush of $90 \mathrm{~mL}$ of water followed the $35 \mathrm{~mL}$ sample, and a potable flush of $110 \mathrm{~mL}$ of water followed all other sample volumes. The potable flushes correspond with the potable flush of the system during nominal operations. The volumes for pseudo urine range from $35 \mathrm{~mL}$ to $940 \mathrm{~mL}$. Volumes $\leq 30 \mathrm{~mL}$ and $\geq 1000 \mathrm{~mL}$ do not undergo accuracy measurement and therefore were not incorporated in the validation sequence. The validation sequence can be seen in Table 1 .

Table 1: Validation Sequence

\begin{tabular}{|c|c|c|}
\hline Validation Segment & Volume (mL) & Specific Gravity \\
\hline 1 & 35 & Average \\
\hline 2 & 90 & ddH $_{2} \mathrm{O}$ \\
\hline 3 & 105 & Average \\
\hline 4 & 110 & dd $_{2} \mathrm{O}$ \\
\hline 5 & 215 & Average \\
\hline 6 & 110 & ddH $_{2} \mathrm{O}$ \\
\hline 7 & 580 & Average \\
\hline 8 & 110 & dd $_{2} \mathrm{O}$ \\
\hline 9 & 690 & $\mathrm{Low}$ \\
\hline 10 & 110 & dd $_{2} \mathrm{O}$ \\
\hline 11 & 940 & $\mathrm{High}$ \\
\hline 12 & 110 & dd $_{2} \mathrm{O}$ \\
\hline 13 & 875 & Average \\
\hline 14 & 110 & dd $_{2} \mathrm{O}$ \\
\hline
\end{tabular}

Ground testing was performed to verify the accuracy, cross-contamination, and residual fluid requirements utilizing the validation sequence. After the UMS is deployed on-orbit, the validation will occur and the samples and data will be returned to determine if the calibration of the UMS unit has been altered due to launch and deployment.

\section{B. Cross-Contamination \& Residual Liquid Testing}

Final ground testing was conducted on the UMS flight unit in the summer of 2010 using the validation sequence volumes, as defined in Table 1. Identical pseudo urine solutions were utilized in the ground testing with the same concentrations as defined above. Pseudo urine consists of the following reagents: Urea, Sodium Chloride, Boric Acid, Creatine, L-leucine, L-methionine, and L-lysine. Urea is the constituent with the highest concentration in all three specific gravity ranges of pseudo urine and is used as the tracer between samples. It is known that viscosity impacts the UMS pressure measurements, so low, medium, and high pseudo urine is used to robustly challenge UMS. 


\section{Cross-Contamination Testing}

Cross-contamination volume was calculated by determining the concentration of urea in the distilled water $\left(\mathrm{ddH}_{2} \mathrm{O}\right)$ sample, and then calculating the volume of the pseudo urine sample which must have remained in the UMS in order to produce the amount of urea in the known volume of distilled water sample. To calculate nominal crosscontamination: ([urea] of Sample 2 x volume of Sample 2) / [urea] of Sample 1. Table 2 displays the results of the ground based validation sequence for cross-contamination. The UMS flight unit did not meet the current requirement of $<0.7 \mathrm{~mL}$ of urine residual. The average cross-contamination volume of seven separate urine test cycles in the validation sequence was $0.80 \mathrm{~mL}$, with a range of $0.74 \mathrm{~mL}$ to $0.87 \mathrm{~mL}$. Any remaining volume would be further diluted following the potable flush (residual volume range: $0.59 \mathrm{~mL}$ to 0.98 ) of the subsequent urine void. Based on the cross-contamination study, the impact to the science samples would be negligible and the exceedance was accepted.

Table 2: Cross-Contamination Results

\begin{tabular}{|c|c|c|c|}
$\begin{array}{c}\text { Cross Over Volume } \\
\text { Between Samples }\end{array}$ & $\begin{array}{c}\text { Low Range } \\
\text { Volume }(\mathbf{m L})\end{array}$ & $\begin{array}{c}\text { Mean } \\
\text { Volume }(\mathrm{mL})\end{array}$ & $\begin{array}{c}\text { High Range } \\
\text { Volume (mL) }\end{array}$ \\
\hline \hline 1 and 2 & 0.67 & 0.73 & 0.8 \\
\hline 3 and 4 & 0.78 & 0.85 & 0.92 \\
\hline 5 and 6 & 0.86 & 0.9 & 0.95 \\
\hline 7 and 8 & 0.77 & 0.85 & 0.94 \\
\hline 9 and 10 & 0.85 & 0.91 & 0.98 \\
\hline 11 and 12 & 0.59 & 0.63 & 0.67 \\
\hline 13 and 14 & 0.68 & 0.76 & 0.85 \\
\hline
\end{tabular}

\section{Sources of Error}

There are two potential error sources; analytical error of the urea sample, which is defined by the percentage of the concentration value, and volumetric error $( \pm 2 \%)$ associated with measuring known volumes of pseudo urine and distilled water samples using a volumetric cylinder prior to processing through the UMS. Therefore, the final data is expressed as a range from the "lowest" and "highest" possible value that could be obtained by taking into consideration that combined analytical error of the urea sample and a fixed $2 \%$ error for volume measurement. The "lowest" value is calculated assuming best case scenario for the analytical and volumetric error, and the "highest" value is calculated assuming worst case scenario for the analytical and volumetric error.

\section{Residual Fluid Testing}

Prior to the start of the validation sequence, the UMS undergoes a system purge where $\sim 100 \mathrm{~mL}$ of potable water is flushed through the system to represent a nominal on-orbit condition. Residual fluid volume is determined by comparing the urea concentration in the samples from validation segment \#1 to the starting pseudo urine solution urea concentration. The dilution of urea in the sample occurs due to residual water remaining in the UMS after flushing/draining. Taking into account the propagation of measurement uncertainty, the lowest residual fluid volume measured was $0.15 \mathrm{~mL}$ and the highest residual fluid volume measure was $3.5 \mathrm{~mL}$. The mean average is therefore calculated to be $1.82 \mathrm{~mL}$ of liquid. The ground based validation testing has confirmed that the UMS meets its requirement to be less than $5 \mathrm{~mL}$ of fluid remaining in the UMS between successive users.

\section{Accuracy Testing}

Ground testing was performed on the UMS flight unit to determine if the accuracy of the unit was within the $\pm 2 \%$ void volume for voids between 100 to $1000 \mathrm{~mL}$ and $\pm 10 \%$ for void volumes between $30 \mathrm{~mL}$ to $100 \mathrm{~mL}$. The accuracy parameter verification requires a total of $95 \%$ of the data points for each of the following volumes to be within the required accuracy; $33 \mathrm{~mL}, 50 \mathrm{~mL}, 70 \mathrm{~mL}, 90 \mathrm{~mL}, 101 \mathrm{~mL}, 350 \mathrm{~mL}, 600 \mathrm{~mL}$, and $900 \mathrm{~mL}$. Pseudo urine was utilized for testing in three specific gravities; 1.01, 1.02, and 1.03. A total of 48 data points were taken; 24 data points were analyzed between volumes of 30-90 mL, and 24 data points were analyzed between volumes 101-900 $\mathrm{mL}$.

The test solutions were preheated in a controlled water bath at approximately $37^{\circ} \mathrm{C}$ to emulate body temperature urine. The actual temperatures of each input volume were recorded, and the mass of each aliquot was measured just 
prior to being introduced into the urinal hose of the UMS. The amount of fluid introduced into the UMS was measured as follows; a graduated cylinder of the appropriate size was filled to the approximate target volume, weighed, slowly emptied into the UMS urinal hose, and reweighed to determine the mass actually delivered. Mass was measured using a calibrated laboratory scale accurate to \pm 0.05 grams. The input volumes were then calculated by dividing the measured input mass by the measured density. The error in input volume can be shown to be accurate to approximately $\pm 0.07 \%$ at $250 \mathrm{~mL}$, which is negligible.

\section{Results}

The UMS met the specification of $\pm 10 \%$ accuracy in the volume range 30 -90 $\mathrm{mL}$ but did not meet the specification of $\pm 2 \%$ accuracy in the volume range 101-900 mL. Nine of the twenty four data points for the higher volume range were greater than the $\pm 2 \%$ range. The largest error for an individual measurement actually observed was $3.95 \%$ (4 $\mathrm{mL}$ at $101.0 \mathrm{~mL}$ input), whereas the largest absolute error was $14.5 \mathrm{~mL}$ (-14.5 mL for input of 606.5, relative error $=-2.39 \%)$. The average mean error of the samples between $100 \mathrm{~mL}$ and $1000 \mathrm{~mL}$ was $0.5 \%(1.2 \mathrm{~mL})$ with a standard deviation of $8.05 \mathrm{~mL}$. Although nine of the twenty four data points were not within the $\pm 2 \%$ specification, the verification test data was accepted. Periodically, there are air bubbles that are present in the ground potable water supply line. In the 1-gravitional test set-up, the air bubbles impact the pressure readings and therefore can cause a miss representation in the accuracy results. On-orbit results are expected to be closer to specification since air bubbles will not impact the hydrostatic pressure and therefore the pressure readings. Figure 6 shows the results plotted to compare each point with the required requirement range.

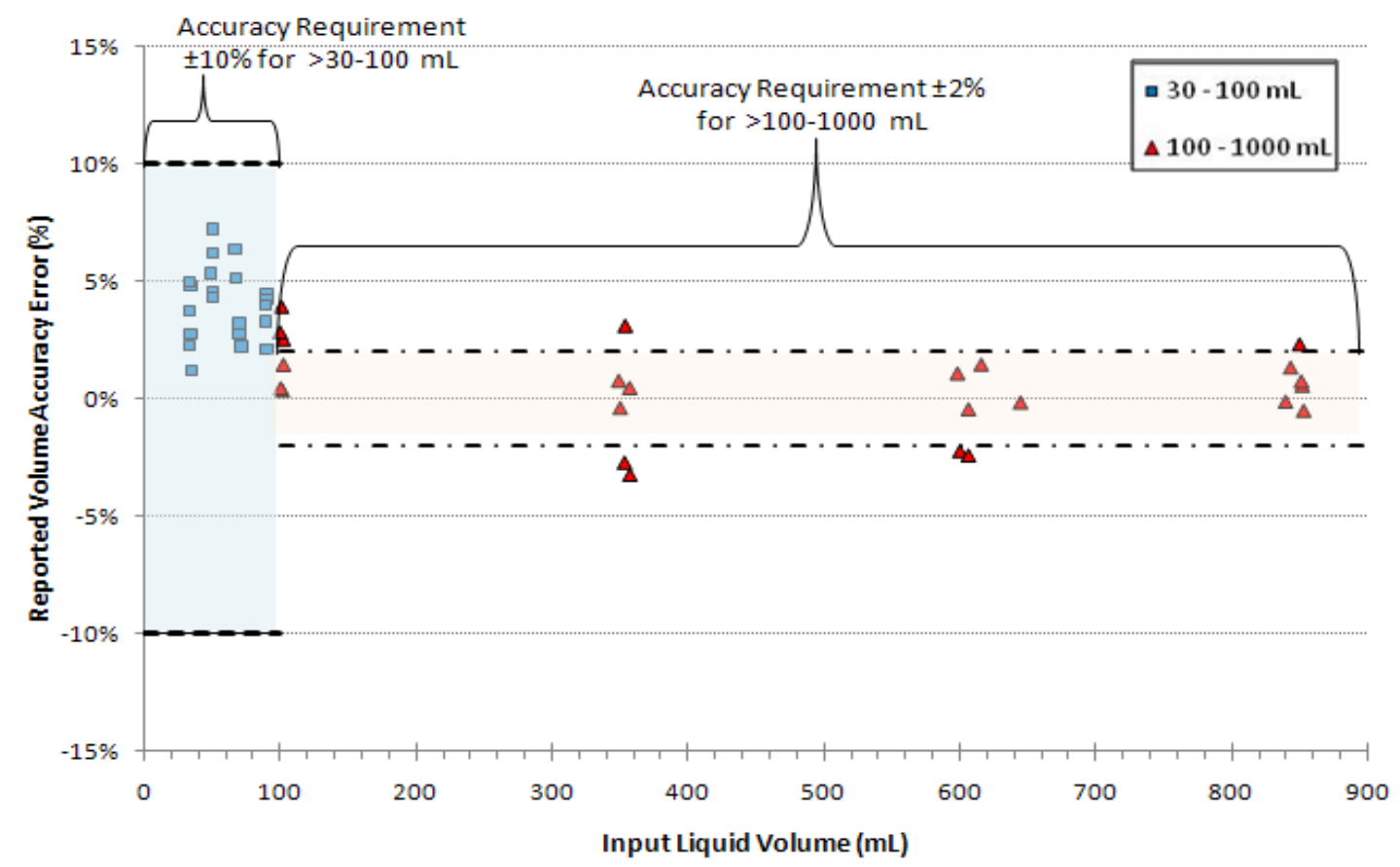

Figure 6: UMS Accuracy Results

\section{WHC Interfaces}

As previously stated, the UMS will interface with the Node 3 WHC integrated rack. The WHC was designed to provide UMS with structural attachments, fluid connections, and electrical power. The UMS and SSC Laptop will use the existing Cabin attachment interfaces for soft mounting. The WHC will provide electrical power and potable water to the UMS in addition to providing urine/air/water disposal. The UMS utilizes the WHC integrated ACY fan which provides airflow in order to draw the fluid (urine/air/water) into the UMS liquid separator. After 
measurements and sampling are complete the urine/air/water from the UMS releases into the WHC. The WHC is certified to operate with or without the UMS. The WHC maintains its own urinal hose and urinal funnel.

The UMS requires the use of the ACY fan for airflow. This activation of the WHC must occur prior to the start of any fluid discharge into the UMS and continue after all liquid has been discharged into the WHC from the UMS. The UMS and the WHC are activated separately using manual switches on each unit.

During the summer of 2010, it was determined that the original location of the urine discharge was no longer feasible with the WHC. The UMS to WHC integration will now attach at the crew member end of the WHC Urinal Hose. A small adapter hose will replace the UMS effluent hose and be utilized to connect the UMS to the WHC urinal hose. Interface airflow testing will be conducted at NASA Johnson Space Center with the WHC training unit and the UMS Engineering Unit to determine the impact of increased pressure drop on flow rate. The airflow testing will determine if the new configuration significantly alters the results obtained during the UMS-ACY testing in September 2009. Concern of increased pressure drop due to the new interface will be evaluated prior to on-orbit use. Figure 6 shows the new configuration of the UMS in the WHC.

The UMS was launched on Space Transportation System (STS) -133 in February 2011. The UMS Adapter Hose is required for the new configuration of UMS to WHC. It is currently scheduled to launch on STS-134 in April 2011. The unit will complete on-orbit validation check-out by utilizing the UMS Validation Kit containing 14 different syringe profiles. Samples from on-orbit validation will be returned home for analysis and testing. Post validation check-out, the UMS will be utilized nominally.

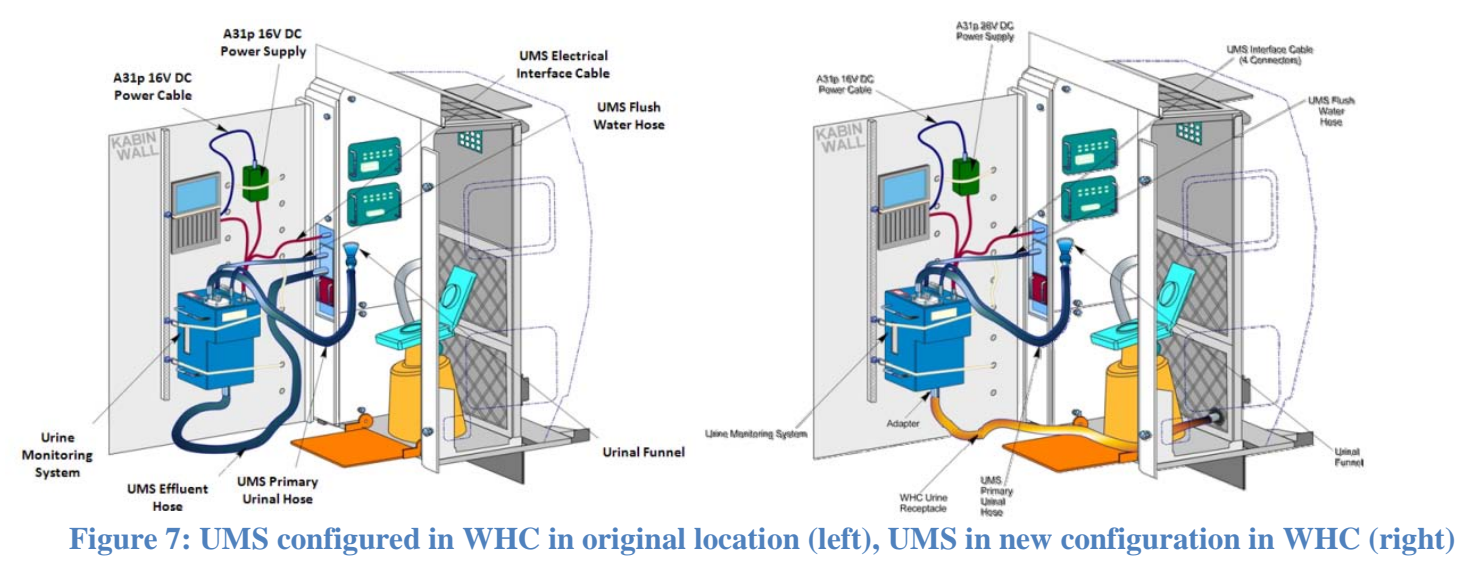

\section{Conclusions}

As demonstrated by the very successful US-Russian joint integration tests of the flight-like UMS and ACY, the two systems are compatible for on-orbit operation of all anticipated void volumes, pressure drops, and repeated operation cycles. The UMS meets its major science requirements (residual liquid) with a few minor excursions (cross-contamination and accuracy). Neither of the excursions are considered detrimental to overall UMS performance. Although there has been a modification of the UMS connection to the ACY urinal hose, it is anticipated that the planned testing will soon validate that approach. UMS will then be able to allow easier, more hygienic, and more frequent monitoring of crew member micturations. The UMS will enable accurate on-orbit urine samples to be obtained and subsequently analyzed. The design and integration of UMS will be a key component to establish an understanding of the impacts that long duration space flight has on the human body.

\section{Acknowledgments}

This paper summarizes the hard work by the numerous National Aeronautics Space Administration (NASA) Johnson Space Center (JSC) and Hamilton Sundstrand project managers, design engineers, and scientists. The NASA JSC team would also like to acknowledge the support and coordination with RSC Energia and NIICHIMMASH. The UMS project is funded by the NASA JSC Life Sciences Office. 


\section{References}

${ }^{1}$ Broyan, J. L., Cibuzar, R. A., “Development of an In-line Urine Monitoring System for the International Space Station,” 2009-01-2400, 39 ${ }^{\text {th }}$ International Conference on Environmental Systems, SAE International, Warrendale, PA, 2009.

${ }^{2}$ Link, D.E., Broyan, J.L., Philistine, C., Balistreri, S.F., ”International Space Station USOS Waste and Hygiene Compartment Development,” 2007-01-3101, 2007 International Conference on Environmental Systems, Chicago, IL.

Link, D., “International Space Station USOS Waste and Hygiene Compartment Development,” 2008-01-2137, 2008 International Conference on Environmental Systems, San Francisco, CA. 